Mulheran, P.A. (2005) Theory of spatial and size scaling in strain-free island ripening. Europhysics Letters, 71 (6). pp. 1001-1007. ISSN 0295-5075

http://strathprints.strath.ac.uk/8494/

Strathprints is designed to allow users to access the research output of the University of Strathclyde. Copyright (C) and Moral Rights for the papers on this site are retained by the individual authors and/or other copyright owners. You may not engage in further distribution of the material for any profitmaking activities or any commercial gain. You may freely distribute both the url (http://strathprints.strath.ac.uk) and the content of this paper for research or study, educational, or not-for-profit purposes without prior permission or charge. You may freely distribute the url (http://strathprints.strath.ac.uk) of the Strathprints website.

Any correspondence concerning this service should be sent to The Strathprints Administrator: eprints@cis.strath.ac.uk 


\title{
Theory of spatial and size scaling in strain-free island ripening
}

\author{
P. A. Mulheran \\ Department of Physics, University of Reading - Whiteknights \\ PO Box 220, Reading RG6 6AF, UK
}

PACS. 81.15.Aa - Theory and models of film growth.

PACS. 81.10.Aj - Theory and models of crystal growth; physics of crystal growth, crystal morphology and orientation.

PACS. 68.55.Ac - Nucleation and growth: microscopic aspects.

\begin{abstract}
A new theory for the ripening of two-dimensional strain-free islands limited by interfacial kinetics is presented. This theory supersedes the mean-field approach by including the environmental contribution to the islands' growth law. Consequently, it accounts not only for the development of the island size distribution; it also explains the concomitant spatial ordering of the islands. The scale invariance of these properties, as observed experimentally and in Monte Carlo simulations, evolves naturally from the theory. Good agreement between the theory and simulation is found. The work is significant for nanostructure production where island spatial and size distributions are key ingredients.
\end{abstract}

Island growth theory has recently been revolutionised by the understanding of the spatial correlations between islands that develop during vapour deposition and its impact on growth rates $[1-6]$. In this paper we consider the spatial correlations that develop regardless of starting conditions [7] through island ripening, that is island growth without vapour deposition. Island ripening is an important process for the fabrication of many nanostructures [8]. To harness the potential for technology, good understanding and control of the spatial arrangements and sizes of ripened islands is essential $[9,10]$.

While some recent studies use strain effects to control the ripening [11,12], the basic understanding of strain-free ripening is not yet fully developed [13]. For example, mean-field theory for ripening has been largely successful for the island size distribution (ISD) [14-18], but this approach cannot account for the scale-invariant spatial ordering found experimentally [19] and in strain-free ripening simulations [20]. In recent work we showed how this spatial ordering arises as a consequence of a "Paedophagous Effect", and how the spatial ordering leads to improved understanding of the ISD [20]. Elements of this effect, leading to the concepts of the "screening length" and "local ripening", have been considered elsewhere in the literature [21,22]. However, we are not aware of any previous theory for the scaling properties of the observed spatial order. In this paper we will build on our earlier approach and provide a theory for the development of both the ISD and the spatial order into a scale-invariant state. 


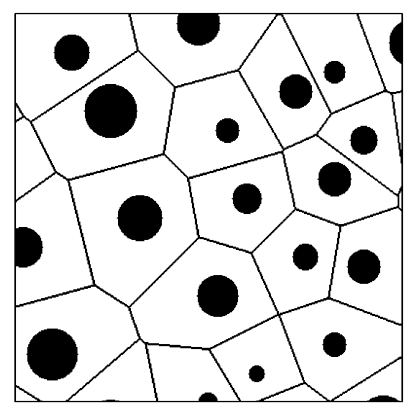

Fig. 1 - Snapshot of a ripened island array with the Voronoi-type edge-cells (VECs) superposed.

In [20] a kinetic Monte Carlo simulation of two-dimensional strain-free island ripening limited by interfacial kinetics was studied. In this minimal model the islands are assumed to be immobile and to vary size while maintaining perfectly circular shape. Upon each iteration, a randomly selected island releases one monomer which diffuses upon a square lattice until it impinges upon any island whereupon it is absorbed. This form of interface-limited ripening kinetics (the monomer diffusion is taken to be much faster than the monomer release rate) $\left({ }^{1}\right)$ corresponds to the classic Wagner model [23,24] where the average island size grows linearly in time $[9,10]$. Despite its simplicity, the model develops the scaling ISD and the scaleinvariant spatial ordering observed experimentally [19]. This spatial ordering emerges even if the monomer release rate is size dependent [20], and we do not expect other features of the model such as treating monomers and islands of size one differently, or forbidding new island nucleation, to have any significant effect on the asymptotic scale invariance.

In this paper we shall first quantify the spatial order using Voronoi-type edge cells (VECs) for each island, where a VEC is defined as the region of substrate closer to that island's edge than to any other island's edge. Figure 1 shows some islands and their VECs at a late stage of simulated ripening. We quantify the network of VECs through its shape probability distribution $f(n, t)$, which is the probability that a randomly selected cell has $n$ sides at time $t$, since the cell shape affects the island growth rates as we describe below. The variance of this shape distribution $\sigma^{2}(t)=\sum_{n}(n-6)^{2} f(n, t)$ evolves over time as shown in fig. 2, where the simulation started with islands placed on a triangular lattice so that all VECs had the same size and shape $\left(f(n, 0)=\delta_{n, 6} \Rightarrow \sigma^{2}(0)=0\right)$. The variance rises rapidly from zero to an asymptotic value $\sigma^{2} \approx 1.23$, following this value over an order of magnitude of simulated time. This asymptotic scale invariance of the shape distribution quantifies the spatial ordering that develops in an entirely self-organised way during ripening. The asymptotic VEC shape probability distribution is also shown in fig. 2, and, along with its scale invariance, is the prime quantity that we wish to explain in the theory developed below.

In a purely mean-field theory the average growth rate of an island depends only on its scaled size:

$$
\frac{\mathrm{d} s}{\mathrm{~d} t}=\left(\frac{r}{\langle r\rangle}-1\right) .
$$

Here time $t$ is scaled by the monomer release rate, $r$ is the island radius and $\langle r\rangle$ is the distribu-

\footnotetext{
$\left({ }^{1}\right)$ The question of whether the model ripening is interface- or diffusion-limited has arisen. In the model the inter-island diffusion time is infinitely faster than the time between monomer releases. In atomistic terms the activation energy for monomer release is therefore infinitely larger than the monomer diffusion activation energy, an appropriate approximation for low temperature ripening. This is why the model is best described as interface-limited ripening.
} 


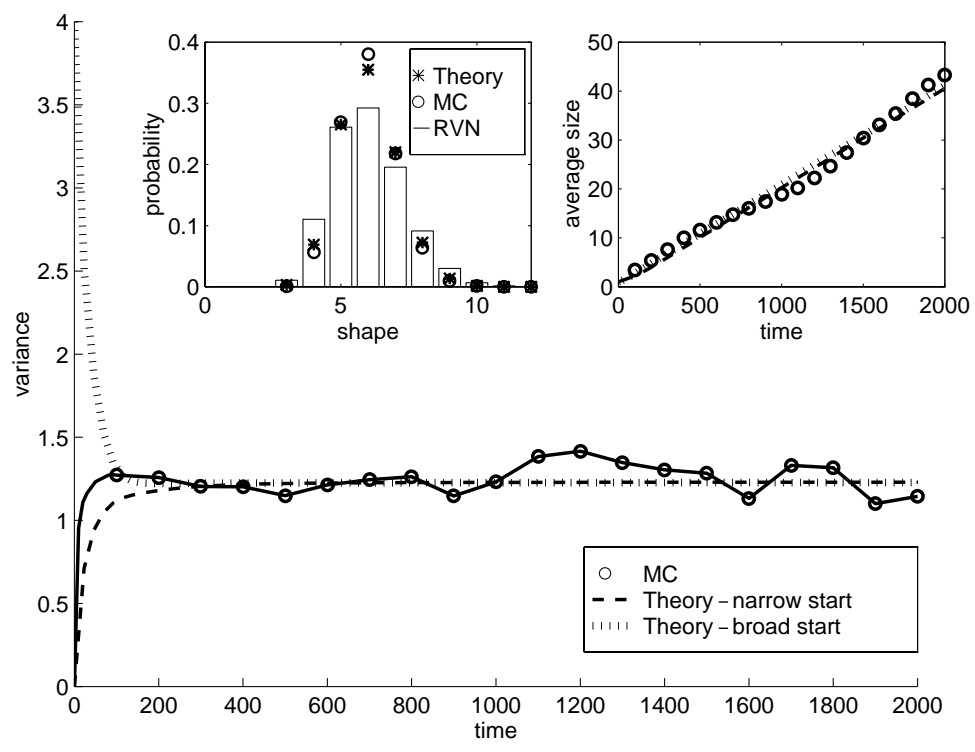

Fig. 2 - The variance of the VEC shape distribution as a function of time. The right inset shows the development of the average island size over time, and is used to calibrate the continuity equation timescale using a single scale factor. The left-hand inset shows the normalised shape distributions from the theory, simulation and a random Voronoi network.

tion average that grows as $t^{1 / 2}$, and $s=\pi r^{2}$ is the two-dimensional island size. Equation (1) comprises the size-independent release rate of the island and its geometric cross-section to capture diffusing monomers in competition with all other islands. The solution of the continuity equation for the island size distribution with eq. (1) yields the Wagner scaling solution $[9,20,23,24]$.

Here we extend this approach beyond the mean field to include environmental effects on island growth rates. The shape of an island's VEC indicates how many first-shell neighbours it has. Some of the monomers emitted by the neighbours will be absorbed by the island, so following our previous work [20] eq. (1) should be modified. The growth rate of an island with an $n$-sided VEC becomes

$$
v(n, s, t)=\frac{\mathrm{d} s}{\mathrm{~d} t}=\frac{n r}{\langle n r\rangle}-1 .
$$

To utilise this growth law equation (2), we construct the continuity equation for $f(n, s, t)$ which is the number of islands with VEC-shape $n$ and size $s$ at time $t$ :

$$
\frac{\partial}{\partial t} f(n, s, t)+\frac{\partial}{\partial s}[v(n, s, t) \cdot f(n, s, t)]=S(n, s, t) .
$$

The term $S(n, s, t)$ on the right-hand side of eq. (3) represents the changes to $f(n, s, t)$ caused by disappearing islands that have shrunk from size one to size zero. When an island disappears, so does its VEC and this causes its neighbouring cells to lose or gain sides. This mixes the various $n$-dependent solutions $f(n, s, t)$, and so in order to complete the model for evolution we must construct a model for $S(n, s, t)$.

Topological changes in a network of triple junctions when cells disappear during soap froth evolution have previously been studied [25]. When a 3-sided cell disappears, all three 

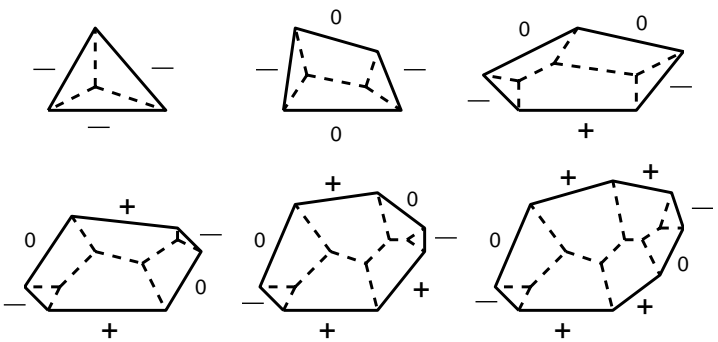

Fig. 3 - The topological rearrangements caused by the removal of variously shaped cells $(n=3-8)$ in a triple-junction network. The solid lines represent the cell being removed, and the dotted lines represent the sides that replace it. The symbols $-/+/ 0$ indicate how these changes affect the shape of the neighbouring cells, i.e. whether they lose or gain a side or stay the same shape.

neighbours lose one side each. When a 4-sided cell vanishes, two neighbours lose one side and two stay unchanged. When a 5-sided cell vanishes, two neighbours lose one side, one neighbour gains one side, and two neighbours stay the same. This is illustrated in the top row of fig. 3. Now in soap froth evolution, driven by the curvature of the cell walls, only 3-, 4-, or 5-sided cells can shrink [25]. However, in our model of island ripening, higher-sided cells may also vanish if the island is much smaller than the average (see eq. (2)), and so we must generalise the topological rules. In the top row of fig. 3 we see how disappearing cells are replaced with chains of triple junctions with the appropriate number of outward arms. Similar chains of triple junctions with $n$ outward arms replace disappearing $n$-sided VECs in general. However, it is not obvious how the chains of triple junctions will be arranged. One straightforward assumption is illustrated in the bottom row of fig. 3, where the chains are extended from one shape to the next by adding triple junctions at $-/ 0$ edges. Also shown is the effect this has on the shapes of the remaining neighbours. This leads to the following generalised rule: when an $n$-sided cell $(n \geq 6)$ disappears, two neighbours lose one side each, two remain the same shape, and $(n-4)$ neighbours gain one side each. These topological rules preserve the average cell shape at six as required by Euler's law for a network of triple junctions [25] and so act as a suitable minimal model for this work.

Before proceeding further we test the utility of this extended topological rule by simulating random Voronoi networks (i.e. networks of Voronoi cells generated in a plane from a twodimensional Poisson process) and counting how many neighbours gain or lose sides when the variously shaped cells are removed. The results are shown in fig. 4(a) which shows that the topological rule does apply reasonable well, but the discrepancy grows with the number of sides on the removed cell. The origin of this discrepancy is deviation in the connectivity from those illustrated in fig. 3, so that a neighbour occasionally gains 2 sides. Nevertheless, the rule as described above works sufficiently well for the purpose of this paper.

There is another fundamental difference between the topological changes that occur during soap froth evolution and those arising in the VEC networks as islands disappear. Many successful models for soap froth evolution neglect correlations between the shapes of neighbouring cells, and assume that a cell is a neighbour of another cell with a probability proportional to its number of sides [26]. However, it would be wrong to extend this idea to the present model by assigning side-gain and loss events (caused by the disappearance of an island) to remaining cells simply in proportion to the number of sides each cell has. In fact cells with large number of sides $n$ are more likely to lose a side when a neighbour disappears, since the average internal angle of the cell $\pi-2 \pi / n$ is larger the greater $n$ is. The sides adjoining the one shared with a disappearing cell are easily extended to connect at a triple point which 

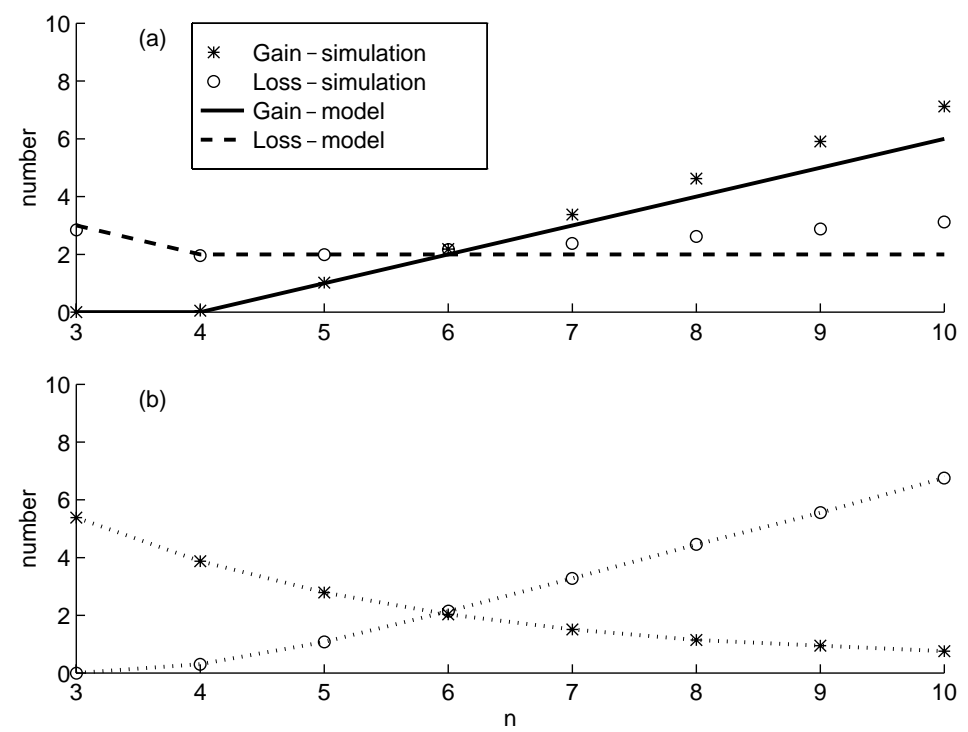

Fig. 4 - (a) The average number of side gains and losses caused to neighbours by the removal of the different shaped cells; (b) the average number of sides gained and lost by different shaped cells when another cell is removed. The data are drawn from a random Voronoi network where each cell is removed in turn. The lines in part (b) are guides to the eye only.

translates to the large- $n$ cell losing a side. Conversely, large- $n$ cells are unlikely to gain sides when neighbours disappear. In a similar fashion, small- $n$ cells are likely to gain a side, but not lose one, when a neighbour disappears. This effect is confirmed in fig. 4(b), where the average number of side gains and losses by the various $n$-sided cells in the random Voronoi network simulation are shown.

An explicit form for $S(n, s, t)$ on the right-hand side of eq. (3) can now be given:

$$
S(n, s, t)=\sum_{m=3}-\frac{\partial f(m, 1, t)}{\partial t}\left[N_{m}^{A} P_{n-1}^{G} f(n-1, s, t)+N_{m}^{R} P_{n+1}^{L} f(n+1, s, t)\right]
$$

In eq. (4) the summation includes the rate at which $m$-sided cells disappear, the number $N_{m}^{A / R}$ of sides each disappearance causes to be added/removed on neighbours (fig. 4(a)), and the probability $P_{n \mp 1}^{G / L}$ that a cell shape $n \mp 1$ is selected to gain/lose a side (from fig. 4(b)).

Equations (2)-(4) are solved numerically for a set of $f(n, s, t)$ with ranges $n=\left[3, n_{M}\right]$ and $s=\left[1, s_{M}\right]$, where $n_{M}$ and $s_{M}$ are made sufficiently large to conserve the total number of monomers in the islands. In implementing the scheme, 3-sided cells cannot lose a side since 2 -sided cells are forbidden in triple-junction networks, and $n_{M}$-sided cells cannot gain sides. It is found that $n_{M}=20$ and $s_{M}=10000$ are sufficient in practice.

As expected for this type of system where there is only length scale, namely the average island size $\langle s\rangle, f(n, s, t)$ evolves rapidly to a scale-invariant form. This is demonstrated in fig. 2 where the variance of the shape distribution $f(n, t)=\sum_{s} f(n, s, t)$ is plotted alongside that from the Monte Carlo simulation. The timescale of the rate equation is matched onto that of the MC simulation using a single scale-factor through comparison of the average island size as shown in the right-hand inset. (In the MC simulation, once released from its parent the average monomer escape probability falls over time, whereas it is convenient to use a 

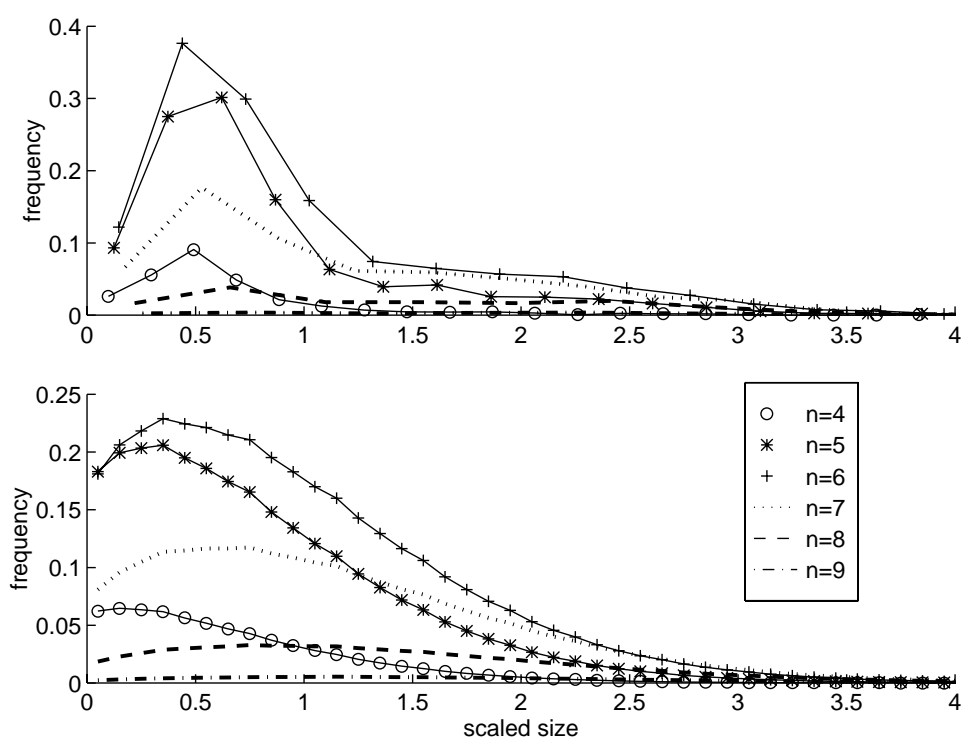

Fig. 5 - The asymptotic scale-invariant distributions $f(n, s, t)$ from the simulation (upper) and theory (lower). The functions are normalised so that $\sum_{n, s} f(n, s, t)=1$. The island size $s$ is scaled to the average $\langle s\rangle=\sum_{n, s} s f(n, s, t)$ for the whole set of distributions.

constant escape rate in the theory). The theory agrees very well with the simulation data, again illustrating that the dynamics of the evolution, now embodied in eqs. (2)-(4), draw the system to an asymptotic scale-invariant state. With the theory it is also easy to test whether the scaling state depends on the starting configuration. The "narrow" solutions start with a triangular distribution of island sizes in range $s=[1,20]$, all with $n=6$ to mimic the simulation data. However starting from a "broad" initial state, where cells have a rectangular distribution in the range $n=[3,9]$, yields an indistinguishable asymptotic scaling state.

The asymptotic shape distribution from the theory is also shown in fig. 2 and is in excellent agreement with the simulation data. It is worth emphasising that the VEC shape distribution is an entirely spatial, non-mean-field property. For comparison in fig. 2 we show the cell shape distribution in a random Voronoi network [27], which has a higher variance of 1.78. The theory presented here therefore predicts for the first time that the spatial arrangements of the ripened islands should be scale-invariant and non-random. This is just the behaviour observed experimentally [19] and in our Monte Carlo simulations [20], only here it is more natural to quantify the spatial ordering in terms of the shapes of the VECs rather than nearest and next-nearest neighbour separations $[19,20]$.

In fig. 5 the asymptotic $f(n, s, t)$ curves found in the simulation and the theory are shown. The theory clearly reproduces the trends in the MC data; however the later curves tend to be more peaked with lower intercepts at small size. The discrepancy in the curves is most probably due to the granular nature of the very small islands in the simulation. Islands can only take integer sizes, and furthermore their radii are also integer since they are imposed on the square mesh for the purposes of simplifying the monomer random walks; modelling size and radius as continuous variables is therefore increasingly crude the smaller the island becomes. However, despite some limitations of the continuum approach adopted in this paper, it successfully predicts the spatial aspects of the emergent ordering during ripening. 
In summary, we have developed a theory for the spatial ordering observed in island ripening. In this theory, an island's growth rate depends on its VEC shape (or number of first-shell neighbours) as well as its radius. Islands with many neighbours are more likely to lose one when another island shrinks, whilst those with few neighbours shrink more rapidly. The dynamics thus draw the distribution of VEC shapes into a peaked, scale-invariant form independent of starting conditions. In this way the theory naturally explains the scale invariance of the spatial ordering. Experimentally, this has been demonstrated using the distribution of first and second nearest-neighbour separations [19], which our Monte Carlo simulations reproduce [20], and so it is suggested that experimental data should also be analysed in terms of cell-shape distributions as a natural measure of spatial order.

\section{REFERENCES}

[1] Mulheran P. A. and Robbie D. A., Europhys. Lett., 49 (2000) 617.

[2] Evans J. W. and Bartelt M. C., Phys. Rev. B, 63 (2001) 235408.

[3] Amar J. G., Popescu M. N. and Family F., Phys. Rev. Lett., 86 (2001) 3092.

[4] Zangwill A., Nature, 411 (2001) 651.

[5] Evans J. W. and Bartelt M. C., Phys. Rev. B, 66 (2002) 235410.

[6] Mulheran P. A., Europhys. Lett., 65 (2004) 379.

[7] Bennett R. A., Tarr D. M. and Mulheran P. A., J. Phys. Condens. Matter, 15 (2003) S3139.

[8] For example, see Kotrla M., Papanicolaou N. I., Vvedensky D. D. and Wille L. T. (Editors), Atomistic Aspects of Epitaxial Growth, NATO Sci. Ser. II, Vol. 65 (Kluwer International Publishers, London) 2002.

[9] Theis W., Bartelt N. C. and Tromp R. M., Phys. Rev. Lett., 75 (1995) 3328.

[10] Bartelt N. C., Theis W. and Tromp R. M., Phys. Rev. B, 54 (1996) 11741.

[11] Liu F., Li A. H. and Lagally M. G., Phys. Rev. Lett., 87 (2001) 126103.

[12] Gai Z., Wu B., Pierce J. P., Farnan G. A., Shu D., Wang M., Zhang Z. and Shen J., Phys. Rev. Lett., 89 (2002) 235502.

[13] Zinke-Allmang M., Thin Solid Films, 346 (1999) 1.

[14] Lifshitz I. M. and Slyozov V. V., J. Phys. Chem. Solids, 19 (1961) 35.

[15] Marder M., Phys. Rev. A, 36 (1987) 858.

[16] Rogers T. M. and Desai R. C., Phys. Rev. B, 39 (1989) 11956.

[17] Ardell A. J., Phys. Rev. B, 41 (1990) 2554.

[18] Marqusee J. A., J. Chem. Phys., 81 (1984) 976.

[19] Carlow G. R. and Zinke-Allmang M., Phys. Rev. Lett., 78 (1998) 4601.

[20] Tarr D. M. and Mulheran P. A., Phys. Rev. E, 68 (2003) 020602(R).

[21] Shorlin K., Zinke-Allmang M. and Fraser D., Phys. Rev. B, 66 (2002) 165403.

[22] Morgenstern K., Rosenfeld G. and Comsa G., Surf. Sci., 441 (1999) 289.

[23] Wagner C., Z. Elektrochem., 65 (1961) 581.

[24] Meerson B., Phys. Rev. E, 60 (1991) 3072.

[25] Weaire D. and Rivier N., Contemp. Phys., 25 (1984) 59.

[26] Flyvbjerg H., Phys. Rev. E, 47 (1993) 4037.

[27] Mulheran P. A., Philos. Mag. Lett., 66 (1992) 219. 